\title{
DESIGN OF A MULTIVARIABLE NEURAL CONTROLLER FOR CONTROL OF A NONLINEAR MIMO PLANT
}

\author{
StanisŁaw BAŃKA, Pawę DWORAK, Krzysztof JAROSZEWSKI \\ Faculty of Electrical Engineering \\ West Pomeranian University of Technology in Szczecin, 26 Kwietnia 10, 71-126 Szczecin, Poland \\ e-mail: \{pawel.dworak; krzysztof.jaroszewski\}@zut.edu.pl
}

\begin{abstract}
The paper presents the training problem of a set of neural nets to obtain a (gain-scheduling, adaptive) multivariable neural controller for control of a nonlinear MIMO dynamic process represented by a mathematical model of Low-Frequency (LF) motions of a drillship over the drilling point at the sea bottom. The designed neural controller contains a set of neural nets that determine values of its parameters chosen on the basis of two measured auxiliary signals. These are the ship's current forward speed measured with respect to water and the systematically calculated difference between the course angle and the sea current (yaw angle). Four different methods for synthesis of multivariable modal controllers are used to obtain source data for training the neural controller with parameters reproduced by neural networks. Neural networks are designed on the basis of 3650 modal controllers obtained with the use of the pole placement technique after having linearized the model of LF motions made by the vessel at its nominal operating points in steady states that are dependent on the specified yaw angle and the sea current velocity. The final part of the paper includes simulation results of system operation with a neural controller along with conclusions and final remarks.
\end{abstract}

Keywords: MIMO multivariable control systems, nonlinear systems, neural control.

\section{Introduction}

Control of multivariable dynamic nonlinear systems is still the subject of intense study and the source of many unresolved issues. For instance, complex motions and/or complex-shaped bodies moving in the case of ships at the boundary between water and air give rise to hydrodynamic forces dependent in a nonlinear way on velocities and positions, thus causing the floating bodies to become strongly nonlinear dynamic plants.

In general, there are two basic approaches to solve the control problem for nonlinear plants. The first one, called "nonlinear", assumes making synthesis of a nonlinear controller that would meet certain requirements over the entire range of control signals variability (Khalil, 2001; Tomera, 2010; Witkowska et al., 2007). The second approach, called "linear", consists in designing an adaptive linear controller with varying parameters to be systematically tuned up in keeping with changing plant operating conditions determined by system nominal operating points (Åström and Wittenmark, 1995; Zhai and $\mathrm{Xu}, 2010)$. Such operating points are usually defined in steady states of the plant. Usually, for linear models obtained by linearization of a given nonlinear model, an appropriate identification method should be used to improve the knowledge of parameter values at actual operating points of the ship. If the description of the nonlinear plant is known, then it is possible to make use of systems with linear controllers prepared earlier for possibly all operating points of the plant. Such controllers can create either a set of controllers with switchable outputs from among which one controller designed for the given system operating point (Bańka et al. 2010a; 2010 b) is chosen, or multi-controller structures whose control signal components are formed, for example, as weighted means of outputs of a selected controller group according to Takagi-Sugeno rules, i.e., with weights being proportional to the degree of their membership of appropriately fuzzified areas of plant outputs and/or other auxiliary signals (Tatjewski, 2007).

What all the above-mentioned multi-controller structures (where not all controllers are at the moment utilized in a closed-loop system) have in common is that all controllers employed in these structures must be stable by themselves, in contrast to a single adaptive controller with varying (tuned) parameters (Vidyasagar, 1985). 
Another approach is to use effective Methods of Predictive Control (MPC) employing nonlinear or on-line linearized models of the plant (Maciejowski, 2002; Rawlings and Mayne, 2009; Limon et al., 2005; Qin and Badgwell, 2003). However, in the case of MIMO nonlinear processes such nonlinear control algorithms are too complex for computations to be performed on-line. Such tasks are particularly difficult when additional constraints on the control signals are considered, which demands using some numerical procedures to solve optimization problems with constraints. When a nonlinear description of the plant is not known accurately, predictive controllers employing artificial intelligence, for example, neural networks (Akesson and Tojvonen, 2006; Ławryńczuk, 2010; Chen and Yea, 2002; van der Boom et al., 2005) or modern versatile neuro-approximators (Tzirkel-Hancock and Fallside, 1992; Fabri and Kadrikamanathan, 2001; Pedro and Dahunsi, 2011) can be used.

In the present paper another approach, which could be seen as a kind of combination of the previously mentioned approaches, is presented. In this solution, controller parameters for each operating point are easily and quickly determined by using a set of neural networks given for controller parameters. The neural networks, whose number equals that of controller parameters, are designed in advance on the basis of the modal controller parameters calculated for possibly all operating points of the plant using the "linear" approach. Such nets allow determining parameters not only at all operating points used in design but also between them, generalizing the parameter values to untrained (unknown) values. In this solution, controller parameters are changeable but changing them frequently enough due to changes in operating point produces no impact on the plant outputs. Such a neural controller could be seen as a solution that consists of only one controller with internal functionality of continuously changing parameters. The neural networks could be designed using parameter values of modal controllers (based on the Luenberger observer or the Kalman filter) obtained by four different methods of the "linear" approach (Bańka, 2007; 2012; Bańka et al., 2013).

The first attempt to use this approach was made by Bańka et al. (2011). They used the EigenVectors Method (EVM) for synthesis of modal controllers in time domain to obtain a data source for training the neural controller, only. But the obtained results of synthesis turned out very inconvienient for training neural nets mainly because of very complicated "surface" shapes generated for varying parameters, especially at the ship's velocities near to $V_{s}=$ 0 and/or at course angles corresponding to yaw angles close to $0^{\circ}, 90^{\circ}, 180^{\circ}$ and $270^{\circ}$. Fortunately, using other methods, especially the Polynomial Matrix Equations (PMEs) method, for synthesis of modal controllers gives better results, more convenient for training neural nets for the same data assumed for synthesis without excessive loss of process control quality.

The organization of this paper is as follows. In Section 2 the structure of the proposed control system is presented. In Section 3 we give a mathematical description of the adopted nonlinear control plant chosen as an example to show the design process for the neural controller. In Section 4 we give a survey of results of synthesis for multivariable modal controllers obtained in both time and frequency domains using four methods named the eigenvalues method, the eigenvectors method, the polynomial method and the polynomial matrix equations method. On the basis of obtained 3650 modal controllers, a set of 36 neural nets is trained. The operation of the neural controller found is tested in Section 5 by system simulation with the nonlinear plant model. We end the paper in Section 6 with conclusions.

\section{Description of the proposed control system structure}

The mathematical description of the plant is given in the form of nonlinear state space and output equations:

$$
\begin{aligned}
\dot{\mathbf{x}}(\mathbf{t}) & =\mathbf{f}(\mathbf{x}(t), \mathbf{u}(t), \mathbf{d}(t)), \\
\mathbf{y}(t) & =\mathbf{g}(\mathbf{x}(t)),
\end{aligned}
$$

where $\mathbf{d}(t)$ represents the disturbance. According to the linear approach adopted in the paper, the linearization of the model (1) is performed for the nominal values of the plant state vector $\mathbf{x}_{o}$, control signals $\mathbf{u}_{o}$ and disturbances $\mathbf{d}_{o}$ that can be calculated from the system of nonlinear algebraic equations:

$$
\begin{aligned}
& \mathbf{0}=\mathbf{f}\left(\mathbf{x}_{o}, \mathbf{u}_{o}, \mathbf{d}_{o}\right), \\
& \mathbf{y}_{o}=\mathbf{g}\left(\mathbf{x}_{o}\right) .
\end{aligned}
$$

As a result of linearization, the following linear state-space models are obtained:

$$
\begin{aligned}
& \dot{\mathbf{x}}(t)=\mathbf{A}\left[\mathbf{x}(t)-\mathbf{x}_{o}\right]+\mathbf{B}\left[\mathbf{u}(t)-\mathbf{u}_{o}\right] \\
& \mathbf{y}(t)-\mathbf{y}_{o}=\mathbf{C}\left[\mathbf{x}(t)-\mathbf{x}_{o}\right]
\end{aligned}
$$

Their matrix transfer functions in the $s$-domain $(s \in$ $\mathbb{C}$ ) can be presented in the form of a relatively right prime (r.r.p.) polynomial Matrix Fraction Description (MFD)

$$
\mathbf{T}(s)=\mathbf{C}\left(s \mathbf{I}_{n}-\mathbf{A}\right)^{-1} \mathbf{B}=\mathbf{B}_{1}(s) \mathbf{A}_{1}^{-1}(s) .
$$

The general block diagram of the proposed control system structure for the plant (1) is depicted in Fig. 1. It is a structure with a neural controller, which replaces an adaptive gain-scheduling controller with measured auxiliary signals $\mathbf{x}_{\mathrm{am}}(t)$ that can be any measured signal like some components of the plant state vector 
or their functions. It is commonly known (Åström and Wittenmark, 1995) that adaptation of control systems can be implemented directly or indirectly (through identification of parameters of the plant model), as well as by on-line tuning the values of controller parameters using the above-mentioned auxiliary measured signals $\mathbf{x}_{\mathrm{am}}(t)$. As has been observed (Bańka, 2010a; 2010b; 2013), it can also be realized as a single adaptive controller with stepwise tuned parameter values or as a set of controllers with a common input $\mathbf{e}(t)$ and switchable outputs $\tilde{\mathbf{u}}(t)$. The above control system for the nonlinear MIMO plant with specified set points $\mathbf{y}_{\text {ref }}$ consists of a neural controller designed on the basis of a bank of multivariable linear modal controllers synthesized for possibly all operating points of the plant. Modal controllers being a basis for making up the above structure are designed for different linear plant models obtained for possibly all operating points of the plant.

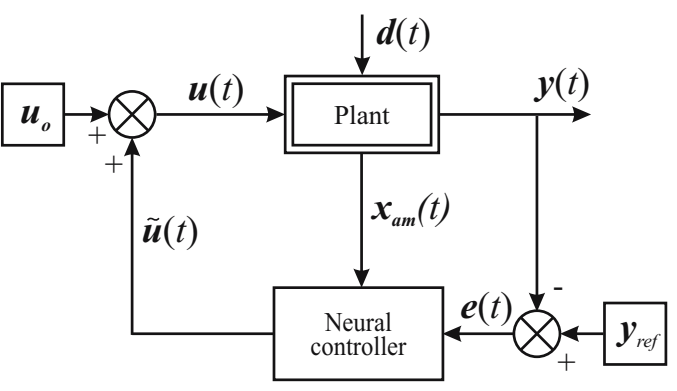

Fig. 1. Block diagram of the general control system structure with an adaptive gain-scheduling neural controller.

In the proposed structure the values of neural gain-scheduling controller parameters determined by a set of neural nets are chosen on the basis of auxiliary variables $\mathbf{x}_{\mathrm{am}}(t)$. During the system operation the incremental values $\tilde{\mathbf{u}}(t)$ generated by the neural controller are added to the nominal values $\mathbf{u}_{o}$ calculated from the Eqns. (2) for each actual nominal value $\mathbf{y}_{o}=\mathbf{y}_{\text {ref }}$.

All synthesized (single) modal controllers are multivariable dynamic systems with (variable) parameters defined in the time domain by the linear state equations

$$
\begin{aligned}
& \dot{\mathbf{x}}_{r}(t)=\mathbf{A}_{r} \mathbf{x}_{r}(t)+\mathbf{B}_{r} \mathbf{e}(t), \\
& \tilde{\mathbf{u}}(t)=\mathbf{C}_{r} \mathbf{x}_{r}(t)+\mathbf{D}_{r} \mathbf{e}(t) .
\end{aligned}
$$

These can be presented in their natural form, which is called "standard", with the following matrices:

$$
\begin{aligned}
& \mathbf{A}_{r}=\mathbf{A}-\mathbf{B F}-\mathbf{L} \mathbf{C}, \quad \mathbf{B}_{r}=\mathbf{L}, \\
& \mathbf{C}_{r}=-\mathbf{F}, \quad \mathbf{D}_{r}=\mathbf{0},
\end{aligned}
$$

where $\mathbf{F}$ is the feedback matrix related to state vector components (reconstructed by the observer) of the plant linear models, and $\mathbf{L}$ is the gain matrix of full order Luenberger observers that reconstruct the state vector of the plant linear models (3). Another possibility (if the polynomial approach with solving polynomial matrix equations is employed) is to present Eqns. (5) in an appropriate canonical form (most common an observable one) with the matrices

$$
\mathbf{A}_{r o}, \mathbf{B}_{r o}, \mathbf{C}_{r o} \text { and } \mathbf{D}_{r}=\mathbf{0}
$$

Unlike the matrices in the "standard" form, they are characterized by a minimal number of parameters different from " 0 " and " 1 ". The above controllers represent strictly causal dynamic systems with $\mathbf{D}_{r}=\mathbf{0}$. In the $s$-domain they are described by strictly proper matrices of rational transfer functions in the form of relatively left prime (1.r.p.) polynomial matrix fractions

$$
\begin{aligned}
\mathbf{T}_{c}(s) & =\mathbf{C}_{r}\left(s \mathbf{I}_{n}-\mathbf{A}_{r}\right)^{-1} \mathbf{B}_{r} \\
& =\mathbf{C}_{r o}\left(s \mathbf{I}_{n}-\mathbf{A}_{r o}\right)^{-1} \mathbf{B}_{r o} \\
& =\mathbf{M}_{2}^{-1}(s) \mathbf{N}_{2}(s),
\end{aligned}
$$

with the following polynomial matrices: $\mathbf{M}_{2}(s) \in$ $\mathbb{R}[s]^{m \times m}$ is the nonsingular row-reduced denominator matrix and $\mathbf{N}_{2}(s) \in \mathbb{R}[s]^{m \times l}$ is the numerator matrix that fulfills the strict inequalities

$$
\operatorname{deg}_{r j} \mathbf{N}_{2}(s)<\operatorname{deg}_{r j} \mathbf{M}_{2}(s), \quad j=1,2, \ldots, m .
$$

The static properties of MIMO modal controllers under discussion depend directly on their gain matrices:

$$
\mathbf{K}_{c}=\mathbf{C}_{r}\left(-\mathbf{A}_{r}\right)^{-1} \mathbf{B}_{r}=\mathbf{M}_{2}^{-1}(0) \mathbf{N}_{2}(0),
$$

and the dynamic properties are determined by poles defined by the eigenvalues of the matrix $\mathbf{A}_{r}$ of each of the controllers, which represent the zeroes of the determinants:

$$
\operatorname{det} \mathbf{M}_{2}(s)=\operatorname{det}\left[s \mathbf{I}_{n}-\mathbf{A}_{r}\right]=0 .
$$

In general, the controllers considered can be stable or unstable. By definition, they cannot exhibit integration properties. In the case under discussion these will be stable multivariable MIMO controllers whose behavior is close to that of PD ones with time lag (Bańka and Latawiec, 2009).

The designed modal controllers were calculated using the following methods: the eigenvalues method, the eigenvectors method, the polynomial method and the polynomial matrix equations method (Bańka, 2012; Bańka et al., 2013). As might be expected, the use of different synthesis methods for modal controllers yielded different results for the same data taken for calculations. The differences in results obtained are fundamental both in terms of construction of neural controllers and also operation quality provided by these controllers in the designed control system. 
As the neural controller approximates parameters of the modal controllers, each of them leads to a stable closed-loop of given dynamics. The so constructed neural controller should yield a stable closed loop for each working point. Thus a fluent change in controller parameters may be regarded as switching between an infinite number of controllers. To show the stability of the above-described closed loop system with a neural (gain-scheduled) controller, the stability theory of nonsmooth systems given by Shevitz and Paden (1994) and used successfully, e.g., by Lee et al. (2001) may been utilized.

\section{Description of the control system for a drilling vessel}

The designing process of the neural modal controller will be exemplified by the controller synthesized for a positioning control system for the MIMO nonlinear dynamic model of the drillship Wimpey Sealab (Wise and English, 1975). The adaptive control system structure considered is studied by means of a 3DOF nonlinear mathematical model of a ship's slow-varying motions described in detail by Bańka and Latawiec (2009) as well as Bańka (2010a; 2010b; 2013). The yaw angle and the ship's position in DSP are defined in an Earth-based fixed reference system whose axes are directed Northwards $(\mathrm{N})$ and Eastwards (E), and whose origin is located over the drilling point on the seabed. In contrast, force and speed components with respect to water are determined in a moving system related with the ship's body with the axes directed to the front and the starboard of the ship with the origin placed in its gravity center. These are shown in Fig. 2.

The mathematical description of the plant is given in the form of nonlinear state space and linear output equations

$$
\begin{aligned}
\dot{x}_{1}= & x_{4} \cos x_{3}-x_{5} \sin x_{3}+V_{c} \cos \Psi_{c}, \\
\dot{x}_{2}= & x_{4} \sin x_{3}+x_{5} \cos x_{3}+V_{c} \sin \Psi_{c}, \\
\dot{x}_{3}= & x_{6}, \\
\dot{x}_{4}= & 0.088 x_{5}^{2}-0.132 x_{4} V_{s}+0.958 x_{5} x_{6} \\
+ & 0.958 u_{1}, \\
\dot{x}_{5}= & -1.4 x_{5} V_{s}-0.978 x_{5}^{3} / V_{s}-0.543 x_{4} x_{6} \\
& +0.037 x_{6}\left|x_{6}\right|+0.544 u_{2}, \\
\dot{x}_{6}= & \left(0.258 x_{5} V_{s}-0.764 x_{4} x_{5}\right. \\
& \left.-0.162 x_{6}\left|x_{6}\right|+u_{3}\right) / a, \\
y_{1}= & x_{1}, \quad y_{2}=x_{2}, \quad y_{3}=x_{3},
\end{aligned}
$$

where state variables $x_{1}, \ldots, x_{6}$ represent the ship position and the course angle over the drilling point as well as her longitudinal, transversal and angular velocities, while $V_{s}=\sqrt{x_{4}^{2}(t)+x_{5}^{2}(t)}$ is the translational velocity of the ship measured with respect to water. The coefficient $a=k_{z z}^{2}+0.0431$ describes the ship's inertia moment together with water associated with the angle motion of the ship around her vertical axis. $k_{z z}^{2}$ is the square of the relative inertia radius referenced to the ship's length $L_{p p}$, and the $V_{c}$ and $\Psi_{c}$ are respectively the velocity and direction of the sea current as indicated in Fig. 2. All the signals appearing in Eqns. (1) are dimensionless, i.e., related to the ship's dimensions and displacement together with the dimensionless time $t=t_{r} / \sqrt{L_{p p} / g} \approx 0.32 t_{r}$.

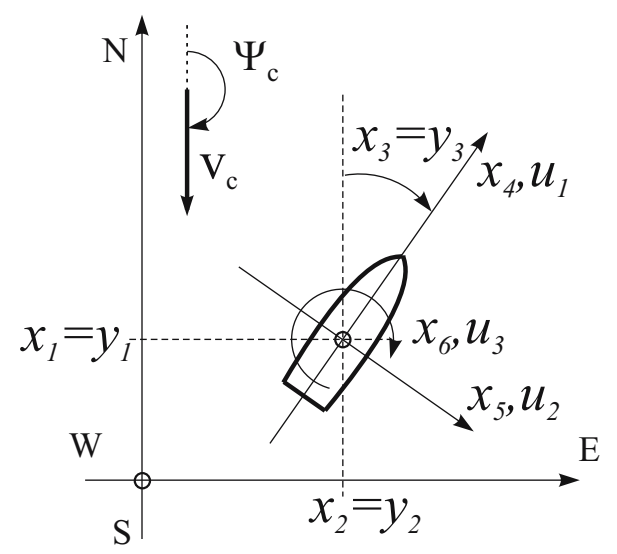

Fig. 2. Ship's co-ordinate systems.

In the proposed structure the controller parameter values are chosen on the basis of auxiliary variables measured. These are the ship's current transitional velocity $V_{s}(t)$ measured with respect to water (it is negative if the ship sails astern, i.e., at $x_{4}(t)<0$ ) and the systematically calculated difference between the sea current angle and the ship's course angle $\Psi_{c}-x_{3}(t)$. The other components of disturbance induced by the wind and wave are omitted here for simplicity of presentation.

The block diagram of the control system for the ship's course and position over the drilling point is depicted in Fig. 3. This system consists of an adaptive (gain scheduling) neural controller with their parameters defined by a set of neural nets chosen during the system operation on the basis of measured auxiliary signals $\mathbf{x}_{a m}$ that are the ship's actual speed $V_{s}$ measured in reference to the water and the systematically calculated difference between the sea current and course (yaw) angles $\Psi_{c}-$ $x_{3}(t)$. For the given plant the nominal values $\mathbf{x}_{o}$ and $\mathbf{u}_{o}$ depend exclusively on the course angle set point $y_{\text {ref }}=y_{30}=x_{30}$, as well as on the velocity and the sea current angle. The additional saturation block visible in Fig. 3. is used to protect against exceeding the maximal values of calculated control signals $\mathbf{u}(t)=\tilde{\mathbf{u}}(t)+\mathbf{u}_{0}$. In a real positioning system (DP) it is usually replaced by the allocation block of calculated control signals $\mathbf{u}(t)$ (components of longitudinal and lateral forces and turning moment) on the main engine and the propellers installed 
on the ship. This is a separate problem that requires application of advanced methods for solution of static optimization tasks with constraints not considered here at all.

As far as the block $\mathbf{u}_{0}$ is concerned, this part of the system in real DP systems may be used for adjusting the values of steady-state errors $\mathbf{e}_{s t} \approx \mathbf{0}$ caused frequently by unmeasured sea disturbances so as to eliminate (or reduce) any deviations resulting in heading and/or position of the vessel in a steady state. If the values of $\mathbf{u}_{0}$ are exactly known, it is theoretically possible to achieve zero steady-state errors $\mathbf{e}_{s t}(t) \rightarrow \mathbf{0}$ together with $\tilde{\mathbf{u}}(t) \rightarrow \mathbf{0}$ (Bańka and Latawiec, 2009). In fact, due to the presence of unmeasured sea disturbances (caused by wind and wave) as well as possible incompatibility with the nominal values $\mathbf{u}_{0}$ and $\mathbf{x}_{0}$ adopted for linearization of Eqns. (12), the steady-state errors will frequently tend to the values $\mathbf{e}_{s t} \neq \mathbf{0}$. Then the output signals of the neural controller will produce (in steady states) values $\tilde{\mathbf{u}}(t) \neq \mathbf{0}$.

In the case of linear models obtained in the form of the state-space equations (3) or the transfer function matrices (4) for the drillship Wimpey Sealab given by nonlinear state-space equations (12) with the effects of wind gusts and wave action having been neglected for clarity, each of the above-discussed synthesis methods leads to yielding strict causal modal full-order controllers described by the space-state equations (5) with matrices $\mathbf{D}_{r}=\mathbf{0}$, which are defined by the strict proper transfer function matrices (8) in the $s$-domain. In order to obtain solutions with the minimal number of parameters whose values are different than " 0 " and " 1 ", the state-space equations for all controllers to be derived will be presented exclusively in canonical forms with matrices (7) in the second Luenberger-Brunovsky canonical observable form:

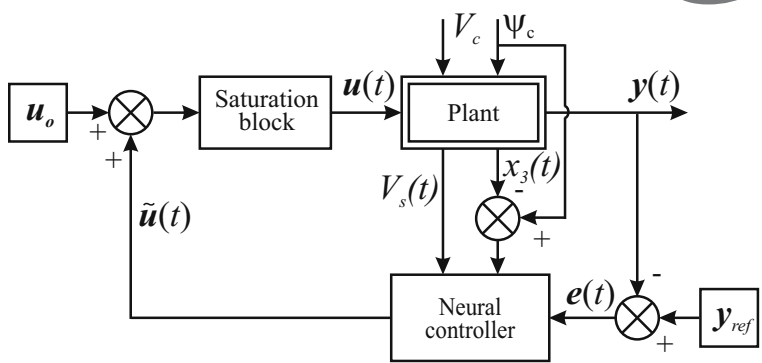

Fig. 3. Block diagram of the proposed control system structure with an adaptive gain-scheduling neural controller for the drilling vessel.

$$
\mathbf{D}_{r o}=\left[\begin{array}{lll}
0 & 0 & 0 \\
0 & 0 & 0 \\
0 & 0 & 0
\end{array}\right] \text {. }
$$

This is a minimal realization (in time domain) of the matrix transfer function (8) when the PME method is used for their synthesis.

They have 36 variable entries: $a_{i j}, i=1,2, \ldots, 6$, $j=2,4,6$, and $b_{i j}, i=1,2, \ldots, 6, j=1,2,3$, dependent on the ship's velocity $V_{s}=\operatorname{sign}\left(x_{4}\right) \sqrt{x_{4}^{2}+x_{5}^{2}}$ and on deviations of the ship's yaw angle $y_{30}=x_{30}$ from the sea current angle $\Psi_{c}$. The controllers have been synthesized for velocities lying in the range $V_{s} \in[-4.9 \div 4.9]$ knots with the resolution of 0.2 knot over the entire range of the round angle, that is, over the range $\Psi_{c}-x_{30} \in\left[0 \div 360^{0}\right]$ with the resolution of $5^{0}(0.0873 \mathrm{rad})$, for the adopted ship relative "radius of gyration" $k_{z z}=1 / 4$. Thus values of each parameter $a_{i j}, b_{i j}$ may be stored in matrices

$$
\mathbf{a}_{i, j}=\left[\begin{array}{ccccc}
a_{i j_{1,1}} & \cdots & a_{i j_{1, l}} & \cdots & a_{i j_{1,73}} \\
\vdots & \ddots & \vdots & & \vdots \\
a_{i j_{k, 1}} & \cdots & a_{i j_{k, l}} & \cdots & a_{i j_{k, 73}} \\
\vdots & & \vdots & \ddots & \vdots \\
a_{i j_{50,1}} & \cdots & a_{i j_{50, l}} & \cdots & a_{i j_{50,73}}
\end{array}\right]
$$

and

$$
\mathbf{b}_{i, j}=\left[\begin{array}{ccccc}
b_{i j_{1,1}} & \cdots & b_{i j_{1, l}} & \cdots & b_{i j_{1,73}} \\
\vdots & \ddots & \vdots & & \vdots \\
b_{i j_{k, 1}} & \cdots & b_{i j_{k, l}} & \cdots & b_{i j_{k, 73}} \\
\vdots & & \vdots & \ddots & \vdots \\
b_{i j_{50,1}} & \cdots & b_{i j_{50, l}} & \cdots & b_{i j_{50,73}}
\end{array}\right]
$$

whose size depends on the number of assumed operating points, here $50 \times 73$. Such generated data were used to train neural networks presented in detail in the next section. For the validation purpose another set of data was also generated. The validation set was obtained for velocities lying in the range $V_{s} \in[-5.05 \div 5.05]$ knots with the resolution of 0.1 knot over the entire range of the 
round angle $\Psi_{c}-x_{30} \in\left[0 \div 360^{0}\right]$ with the resolution of $1^{0}(0.0175 \mathrm{rad})$.

Remark 1. It should be noted that dividing by a signal representing the ship's translational velocity $V_{s}(t)$ with respect to water takes place in the above nonlinear ship motion model. This accounts for indeterminate behavior of the nonlinear model at zero-valued ship velocity, i.e., when dividing by $V_{s}(t)=0$ occurs. The nonlinear ship model (12) loses physical meaning and this has definite consequences not only during the system simulation, but also for the control system synthesis, since linear models become indeterminate at $V_{s}=0$. Such a situation takes place when the ship is carried along by currents or when the ship stands still over the drilling point in calm water at $V_{c}=0$. Such a problem would not exist for neural controller.

\section{Neural controller synthesis}

Due to the complexity of a variety of values of parameters $a_{i j}$ and $b_{i j}$ of the controller described by (13), the proposed neural controller consists of a group of neural networks, each designed for an approximation of a specific parameter $a_{i j}$ or $b_{i j}$ on the basis of multivariable modal controllers obtained for possibly all operating points of the plant. As all networks are independent of each other, the structure of each of them depends on the complexity of a variety of the approximated parameter only. Examples of the variability of the same controller parameters $a_{34}$ of matrix $\mathbf{A}_{r o}$ and $b_{22}$ of matrix $\mathbf{B}_{r o}$ vs. ship's velocity and yaw angle obtained by different methods of synthesis are presented in form of surfaces in Figs. 4 and 5, respectively.

The most promising results of designing neural networks were expected from a method that is characterized by the smoothest surface of the parameter change. At the first glance the smoothest surfaces are obtained using the polynomial matrix equations method. The smoothens of variety of the given parameter $a_{i j}$ and $b_{i j}$ can be also evaluated in a more quantitative way by a factor defined by the following relationship:

$$
\begin{aligned}
s_{i, j}= & \left(\sum_{k=1}^{49} \sum_{l=1}^{73}\left|p_{i, j_{k, l}}-p_{i, j_{k+1, l}}\right|\right. \\
& \left.+\sum_{k=1}^{50} \sum_{l=1}^{72}\left|p_{i, j_{k, l}}-p_{i, j_{k, l+1}}\right|\right) \\
& \times\left(\sum_{k=1}^{50} \sum_{l=1}^{73} p_{i, j_{k, l}}\right)^{-1},
\end{aligned}
$$

where $p_{i, j_{k, l}}$ stands for elements of the matrices $\mathbf{a}_{i, j}$ and $\mathbf{b}_{i, j}$, defined in (14) and (15), respectively. The lowest values of coefficient (16) were achieved for modal controllers calculated using the polynomial matrix equations method.

Considering the complexity of the surfaces, many different types and structures of neural networks were proposed and taken into account during the design process. For each parameter obtained by each method, one specific net was designed. At the very beginning, four types of neural networks were taken into account: feed-forward back propagation, cascade-forward back propagation, radial basis with fewer neurons and radial basis exact fit. Although it is well known that RBF nets are good interpolators or approximators, the main problem is that their size (the number of neurons) in the first (radial) layer is at most equal to the number of training points. As, in our case, we have a big training pattern, we have to give up designing the RBF function due to the lack of memory in the MATLAB environment. The design process was conducted using the MATLAB/Simulink environment. Structures of the back propagation networks designed are presented in Table 1.

All designed back propagation networks have two inputs due to the signals affecting changes in controller parameters, and one output calculated by the net as the current value of the controller parameter. What is more, "tansig" transfer functions were used in all hidden layers and the "purelin" transfer function was used in each output layer; the Levenberg-Marquardt optimization algorithm (TRAINLM) was employed and the "MSE" criterion was employed as a performance function as well. Using a high-end computer, designing such a huge number of back propagation nets $(20$ net structures $\times 36$ controller parameters $\times 4$ synthesis methods $=2880$ nets) took a long time, yet it turned out to be possible.

In searching for the best controller, at the very beginning, the most suitable nets were investigated. It was assumed that the most similar shape and values of the surfaces at known points would be pointed out due to the one of three calculated criteria:

1. The sum of the differences between the values of points on the original surface and those estimated by the net, described by

$$
e_{i, j}=\sum_{k=1}^{50} \sum_{l=1}^{73}\left(p_{i, j_{k, l}}-y_{k, l}^{\mathrm{net}}\right),
$$

where $p_{i j_{k, l}}$ stands for elements of matrices $\mathbf{a}_{i, j}$ and $\mathbf{b}_{i, j}$ while $y_{k, l}^{\text {net }}$ for the values estimated by the net.

2. The sum of the absolute values of the differences between values of the points on the original surface and those estimated by the net, described by

$$
e_{i, j}=\sum_{k=1}^{50} \sum_{l=1}^{73}\left|p_{i, j_{k, l}}-y_{k, l}^{\text {net }}\right|
$$



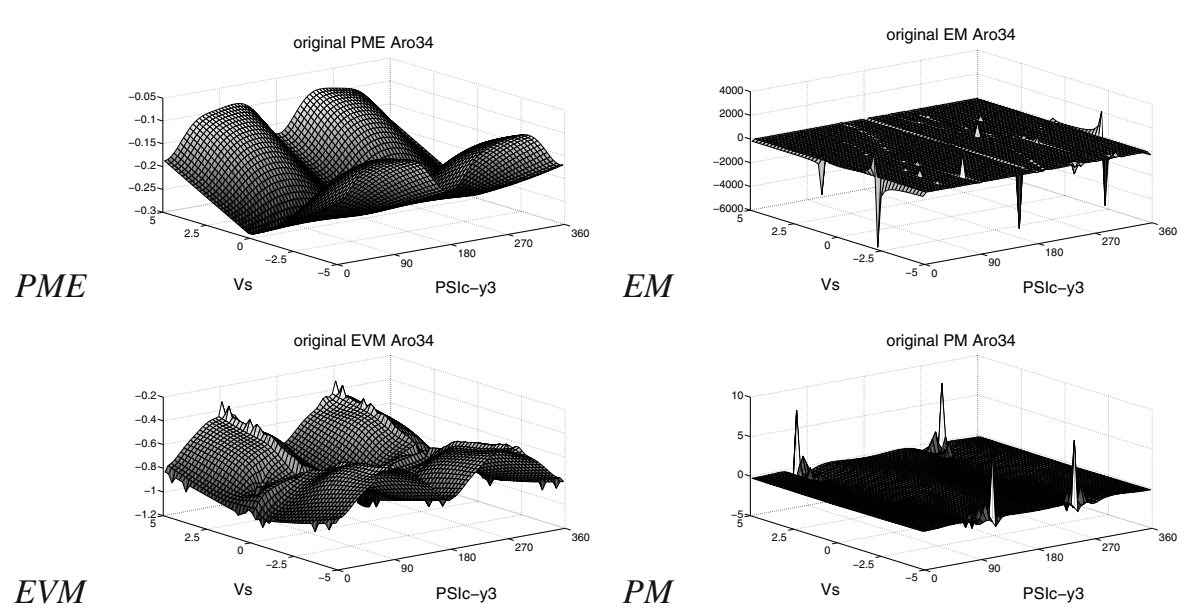

Fig. 4. Variability of the parameter $a_{34}$ of matrix $\mathbf{A}_{r o}$ vs. the ship's velocity and yaw angle obtained by different synthesis methods.
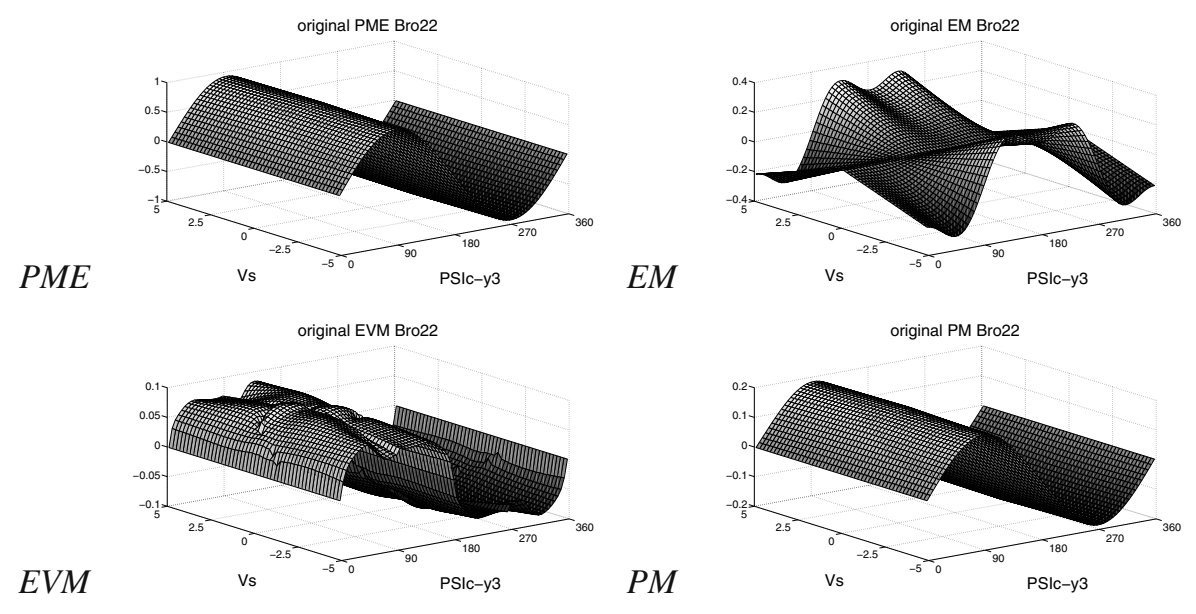

EM

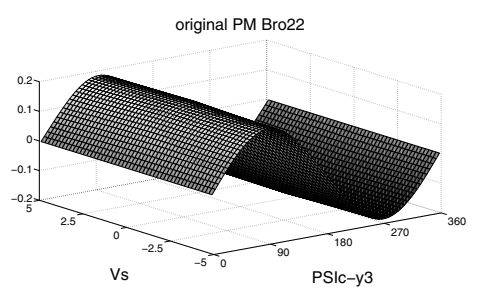

Fig. 5. Variability of the parameter $b_{22}$ of matrix $\mathbf{B}_{r o}$ vs. the ship's velocity and yaw angle obtained by different synthesis methods.

where $p_{i j_{k, l}}$ stands for elements of matrices $\mathbf{a}_{i, j}$ and $\mathbf{b}_{i, j}$ while $y_{k, l}^{\text {net }}$ for the values estimated by the net.

3. The sum of the squared average values of the differences between values of the points on the original surface and those estimated by the net, described by

$$
e_{i, j}=\sum_{k=1}^{50} \sum_{l=1}^{73}\left(p_{i, j_{k, l}}-y_{k, l}^{\text {net }}\right)^{2}
$$

where $p_{i j_{k, l}}$ stands for elements of matrices $\mathbf{a}_{i, j}$ and $\mathbf{b}_{i, j}$ while $y_{k, l}^{\text {net }}$ for values estimated by the net.

What is more, the evaluation of the nets was done by a visual assessment of the obtained surfaces. Using the above criteria, generally, the nets designed on the basis of the data for the PME type of synthesis were pointed out as those whose outputs produce signals most fitted to the original one, which confirms the results obtained using the formula (16).
Selection of specific net structures from among the ones designed for the PME type of synthesis was conducted according to the former numerical criterion. The criterion (17) was taken into consideration as most suitable during the evaluation process. However, the remaining criteria often pointed out at the same structures as the best ones. The summary of error values for the net designed on the basis of PME synthesis is presented in Tables 2 and 3.

In the next two figures (Figs. 6 and 7) a comparison of network responses with the original surface for all synthesis methods exemplified by the parameters $a_{34}$ and $b_{22}$ is presented. As may be noticed, mainly due to their simplicity, the obtained surfaces (produced by neural networks) are most similar to their origins in the case of the PME synthesis method. The differences are almost imperceptible. All surfaces of variable entries of matrices $\mathbf{A}_{\text {ro }}, \mathbf{B}_{\text {ro }}$ versus the ship's velocity $V_{s}$ and yaw angle $\Psi_{c}-x_{30}$ obtained by the PME method have been illustrated in our previous work (Bańka et al., 2013). 
Table 1. Structures of the trained back propagation networks.

\begin{tabular}{|c|c|c|c|c|c|c|}
\hline & & \multicolumn{5}{|c|}{ Number of neurons in the layer } \\
\hline No. & Input & \multicolumn{4}{|c|}{ Hidden } & Output \\
\hline & & (1 $^{\mathrm{st}}$ & $2^{2^{\text {nd }}}$ & $3^{\text {rd }}$ & $4^{\text {th }}$ & \\
\hline 1 & 2 & 3 & & & & 1 \\
\hline 2 & 2 & 6 & & & & 1 \\
\hline 3 & 2 & 9 & & & & 1 \\
\hline 4 & 2 & 12 & & & & 1 \\
\hline 5 & 2 & 15 & & & & 1 \\
\hline 6 & 2 & 18 & & & & 1 \\
\hline 7 & 2 & 6 & 3 & & & 1 \\
\hline 8 & 2 & 9 & 3 & & & 1 \\
\hline 9 & 2 & 9 & 6 & & & 1 \\
\hline 10 & 2 & 12 & 6 & & & 1 \\
\hline 11 & 2 & 12 & 9 & & & 1 \\
\hline 12 & 2 & 15 & 9 & & & 1 \\
\hline 13 & 2 & 9 & 6 & 3 & & 1 \\
\hline 14 & 2 & 12 & 6 & 3 & & 1 \\
\hline 15 & 2 & 12 & 9 & 3 & & 1 \\
\hline 16 & 2 & 12 & 9 & 6 & & 1 \\
\hline 17 & 2 & 15 & 9 & 6 & & 1 \\
\hline 18 & 2 & 15 & 12 & 3 & & 1 \\
\hline 19 & 2 & 15 & 12 & 6 & & 1 \\
\hline 20 & 2 & 12 & 9 & 6 & 3 & 1 \\
\hline
\end{tabular}

Table 2. Summary of error values for elements of $\mathbf{A}_{r o}$.

\begin{tabular}{|l|l|l|l|l|}
\hline Aro net & \multicolumn{1}{|c|}{ Best structure } & \multicolumn{3}{|c|}{ Choice due to the absolute error criterion } \\
\hline \hline No. & $\begin{array}{l}\text { Type and number } \\
\text { of neurons in layers }\end{array}$ & $\begin{array}{l}\text { Value of the } \\
\text { error criterion }\end{array}$ & $\begin{array}{l}\text { Value of the absolute } \\
\text { error criterion }\end{array}$ & $\begin{array}{l}\text { Value of the mean square } \\
\text { error criterion }\end{array}$ \\
\hline 12 & Cascade-forward 12-9-3 & 0.77 & 2.38 & 3.51 e-04 \\
\hline 14 & Cascade-forward 15-12-6 & 0.40 & 2.68 & 3.81 e-04 \\
\hline 16 & Cascade-forward 12-9-6-3 & -0.15 & 0.31 & 3.82 e-06 \\
\hline 22 & Feed-forward 15-12-6 & -0.88 & 5.10 & 1.39 e-03 \\
\hline 24 & Cascade-forward 15-12-6 & -0.37 & 1.39 & 8.83 e-05 \\
\hline 26 & Cascade-forward 9-6 & -0.25 & 0.44 & 9.04 e-06 \\
\hline 32 & Cascade-forward 12-9-6 & -0.30 & 13.75 & 9.25 e-03 \\
\hline 34 & Cascade-forward 12-9-6 & 0.48 & 13.99 & 1.20 e-02 \\
\hline 36 & Cascade-forward 12-9-6 & -0.08 & 1.14 & 7.96 e-05 \\
\hline 42 & Cascade-forward 15-12-6 & -0.39 & 6.45 & 2.37 e-03 \\
\hline 44 & Cascade-forward 15-12-6 & -0.59 & 8.91 & 5.86 e-03 \\
\hline 46 & Cascade-forward 12-9-6-3 & 0.029 & 0.04 & 7.76 e-08 \\
\hline 52 & Cascade-forward 12-9-6-3 & 0.21 & 0.93 & 6.01 e-05 \\
\hline 54 & Cascade-forward 15-12-3 & -1.23 & 2.29 & 2.77 e-04 \\
\hline 56 & Cascade-forward 15-12-6 & -0.42 & 5.08 & 1.37 e-03 \\
\hline 62 & Cascade-forward 12-9-6 & -0.08 & 0.92 & 5.08 e-05 \\
\hline 64 & Cascade-forward 12-9-6-3 & 0.36 & 2.33 & 3.09 e-04 \\
\hline 66 & Feed-forward 15-12-3 & 1.44 e-12 & 1.53 e-11 & 1.121 e-26 \\
\hline
\end{tabular}

All of this makes quite a complex picture of problems connected with implementation of the proposed multi-controller structures of linear modal controllers designed for steady states, but actually utilized to control transients. This is possible, as evidenced below, by results of simulations carried out with the ship's nonlinear model (12) for all obtained sets of modal controllers realized as a single adaptive controller with tuned parameters.

\section{Results of simulation tests}

All simulation tests were carried out without regard to the effect produced by wind and wave action in the presence of sea current of $V_{c}=2$ knots at $\Psi_{c}=180^{\circ}$ with the 
Table 3. Summary of error values for elements of $\mathbf{B}_{\text {ro }}$.

\begin{tabular}{|l|l|l|l|l|}
\hline Bro net & \multicolumn{1}{|c|}{ Best structure } & \multicolumn{3}{|c|}{ Choice due to the absolute error criterion } \\
\hline \hline No. & $\begin{array}{l}\text { Type and number } \\
\text { of neurons in layers }\end{array}$ & $\begin{array}{l}\text { Value of the } \\
\text { error criterion }\end{array}$ & $\begin{array}{l}\text { Value of the absolute } \\
\text { error criterion }\end{array}$ & $\begin{array}{l}\text { Value of the mean square } \\
\text { error criterion }\end{array}$ \\
\hline 11 & Cascade-forward 9-6 & -5.10 e-02 & 6.60 e-02 & 2.03 e-07 \\
\hline 12 & Cascade-forward 9-6 & 8.61 e-03 & 0.18 & 1.19 e-06 \\
\hline 13 & Cascade-forward 12-9-6-3 & -1.38 & 9.62 & 4.82 e-03 \\
\hline 21 & Cascade-forward 15-9-6 & 8.24 e-02 & 2.11 & 3.24 e-04 \\
\hline 22 & Cascade-forward 12-9-3 & 4.19 e-02 & 1.18 & 9.03 e-05 \\
\hline 23 & Cascade-forward 9-6-3 & 0.28 & 2.59 & 3.52 e-04 \\
\hline 31 & Cascade-forward 15-12-6 & 1.91 & 6.59 & 2.10 e-03 \\
\hline 32 & Cascade-forward 12-9-6-3 & 1.44 & 8.88 & 4.44 e-03 \\
\hline 33 & Cascade-forward 15-12-3 & -0.18 & 4.67 & 1.37 e-03 \\
\hline 41 & Cascade-forward 12-9-6-3 & 3.38 e-02 & 10.88 & 6.70 e-03 \\
\hline 42 & Cascade-forward 15-12-3 & -0.62 & 13.03 & 9.66 e-03 \\
\hline 43 & Cascade-forward 15-9-6 & 3.52 e-02 & 0.48 & 1.12 e-05 \\
\hline 51 & Cascade-forward 3 & 0 & 0 & 0 \\
\hline 52 & Cascade-forward 3 & 0 & 0 & 0 \\
\hline 53 & Cascade-forward 15-12-6 & 4.63 & 11.00 & 5.58 e-03 \\
\hline 61 & Cascade-forward 15-12-3 & -0.30 & 4.36 & 1.17 e-03 \\
\hline 62 & Cascade-forward 15-9 & -1.32 & 3.25 & 6.60 e-04 \\
\hline 63 & Cascade-forward 12-9-6 & 2.29 & 6.46 & 2.42 e-03 \\
\hline
\end{tabular}

original PME Aro34

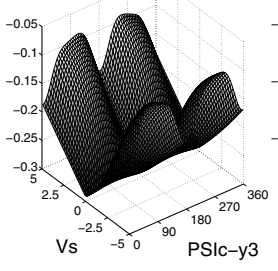

original EVM Aro34

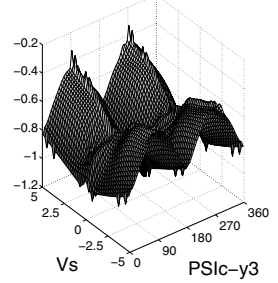

net cf 1296

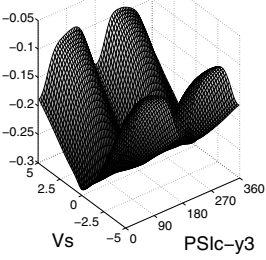

net cf 15123

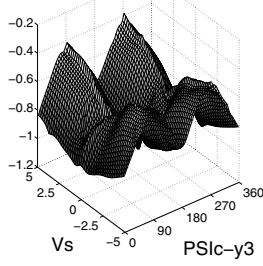

$E M$

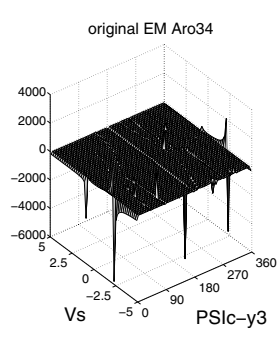

net ff 15123

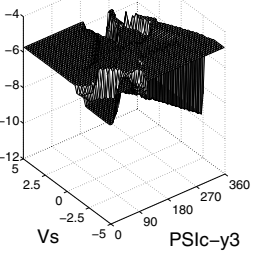

net ff 1296

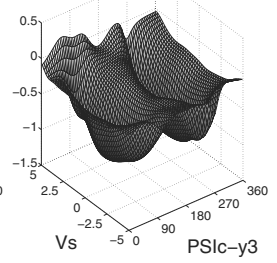

EVM

Fig. 6. Comparison of selected surfaces of the same parameter $a_{34}$ for different methods of synthesis.

use of the ship's nonlinear model (12) that describes slow varying ship motions in $3 \mathrm{DOF}$. All presented results of simulation tests for stepwise tuned controllers and a neural controller were carried out in the Hardware in the Loop (HiL) environment. The HiL test stand consists of two 1104 dSpace R\&D controller boards, which allows one to simulate independently the Wimpey Sealab model (12) and the controller in real time.

The ship's and controller inputs and outputs as well as the current ship velocity were transmitted between cards by wires with the use of 12 bit independent AQ and AI. The maximum sampling period for the described neural controller with the use of the 1104 dSpace R\&D controller board is $100 \mathrm{~ms}$, which is much more than necessary for the ship model given.

Yet the controller has also been tested to work in an industrial PLC controller X20 CP 1585 of B\&R, where its recorded average scan period is $1013 \mu \mathrm{s}$ with the size of the function block 188936 B. For comparison, the Takagi-Sugeno fuzzy controller built on all sets of synthesized 3650 local linear controllers in X20 CP 1585 needs 1686732 B of memory and its average scan period equals $141 \mu \mathrm{s}$. Taking into account the so-called "curse of dimensionality", implementation of 

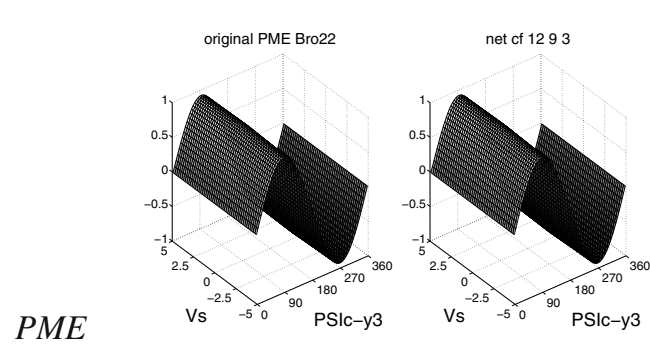

$P M E$
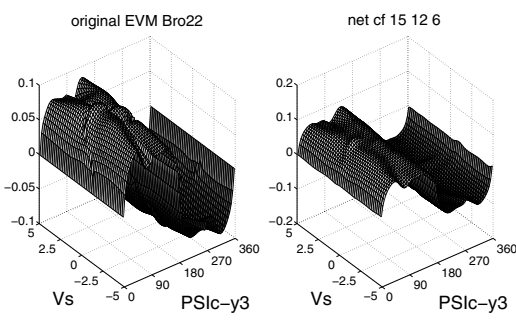

\section{$E M$}
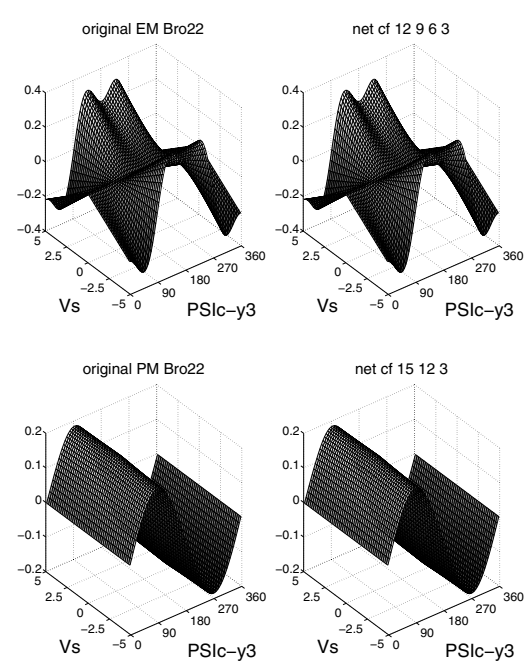

$P M$

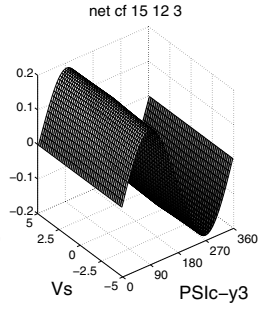

Fig. 7. Comparison of selected surfaces of the same parameter $b_{22}$ for different methods of synthesis.

the fuzzy Takagi-Sugeno MIMO controller for a great number of the auxiliary signals may be difficult (if possible) with its scan periods rapidly rising. The neural controllers described in the paper should not be such space consuming with almost the same scan periods.

The tests were conducted for many different initial states defined by appropriate ship positions, course angles and ship initial velocities. In typical situations, that is, when the ship sailed bow on against the current at $V_{s} \neq$ 0 , all controllers behaved quite correctly yielding time responses without excessive oscillations experienced by the control signals $\mathbf{u}(t)$. In that case the ship could always be brought to the drilling point with the assumed preset course angle, and then she could be moved to any specified position. This is demonstrated by time responses depicted in Fig. 8, obtained with adaptive (stepwise tuned) and neural controllers found by the polynomial matrix equations method.

During the simulations the ship was brought to the drilling point from a position about $100 \mathrm{~m}(r=1)$ distant situated on the left below the drilling point with the adopted initial course angle $x_{3}(0)=35^{0}$ and velocity components $x_{4}(0)$ and $x_{5}(0)$, which corresponds to the ship's sailing bow on against the current $V_{s}(0)=V_{c}=2$ knots. After reaching the drilling point with the specified course angle equal to $y_{3 \mathrm{ref}}=x_{30}=0^{0}$, the course angle was changed to $y_{3 \mathrm{ref}}=x_{30}=60^{\circ}$, and then the reference values were changed stepwise for both ship's position coordinates so that the ship move through a distance of about $100 \mathrm{~m}$ from the right over the drilling point and come to a standstill at a distance of $100 \mathrm{~m}$ with a velocity of $V_{s}=V_{c}=2$ knots relative to water and bringing the ship's course angle $y_{3}(t)=x_{3}(t)$ to the preset value $y_{3 \mathrm{ref}}=x_{30}=60^{\circ}$.

However, more interesting and instructive are responses obtained for atypical situations when the ship sails stern first against the current, especially at changes in the sign of the linear ship velocity $V_{s}$ in the vicinity of $V_{s}=0$. This may happen when the ship is brought to the drilling point with the current (conditioned, for example, by an unfavorable direction of the wind or sea waves being in opposition to the sea current direction $\Psi_{c}$ ) or when changing the ship's yaw angle over the drilling point caused by a change in wind or wave direction.

To investigate the matter, the remaining simulations were performed for the ship situated initially about $100 \mathrm{~m}$ on the left over the drilling point with the initial course angle $x_{3}(0)=125^{0}$ and velocity components $x_{4}(0)$ and $x_{5}(0)$, which corresponds at the beginning of simulations to moving astern at $V_{s}(0)=-V_{c}=-2$ knots. In these simulations, the preset ship course angles $y_{3 \mathrm{ref}}=x_{30}$ over the drilling point, as well as making later changes in the yaw angle and ship final positions, were performed just in the same way as earlier, keeping as far as possible the same time conditions for manoeuvring. Simulation results obtained for this scenario for sets of controllers designed by the polynomial matrix equations method are shown in Fig. 9.

It may bee seen in Figs. 7 and 9 that a neural controller eliminates problems with switching between controllers in a set and makes the control signals smoother. Moreover, there is no need to calculate (search for) the appropriate number of such controller set, which would demand estimation of, e.g., levels of steady-state errors as the neural controller covers all possible working points.

\section{Concluding remarks}

It follows from the simulation tests carried out that the proposed concept of control system realization with the 
Adaptive (stepwise tuned) controller
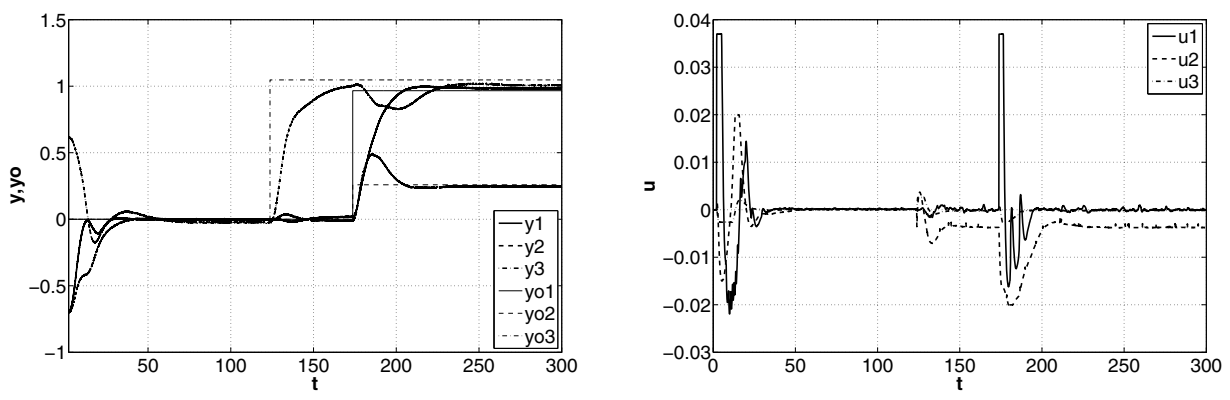

Neural controller
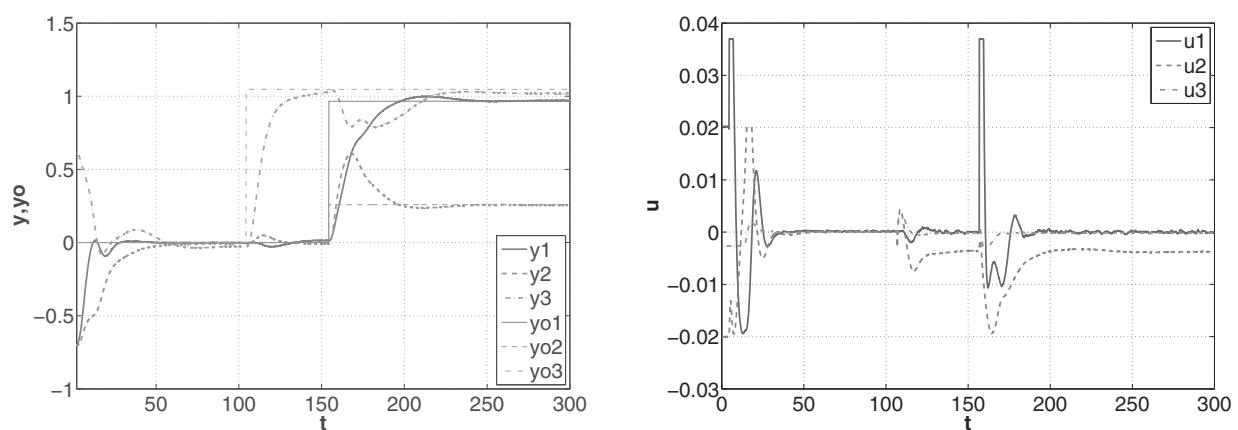

Fig. 8. Plots of the ship's position and yaw angle and control signals $\mathbf{u}(t)$ vs. time in the process of bringing the ship to the drilling point bow on against the current.

Adaptive (stepwise tuned) controller
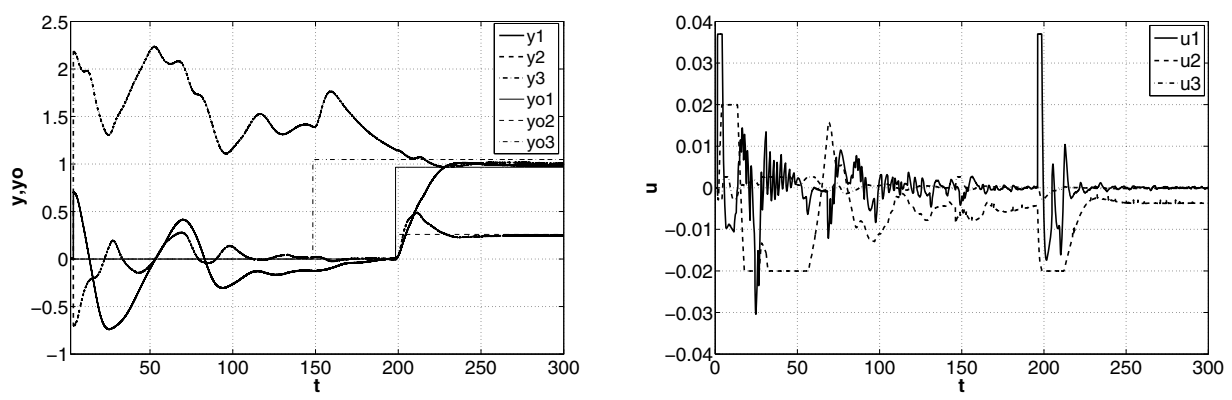

Neural controller
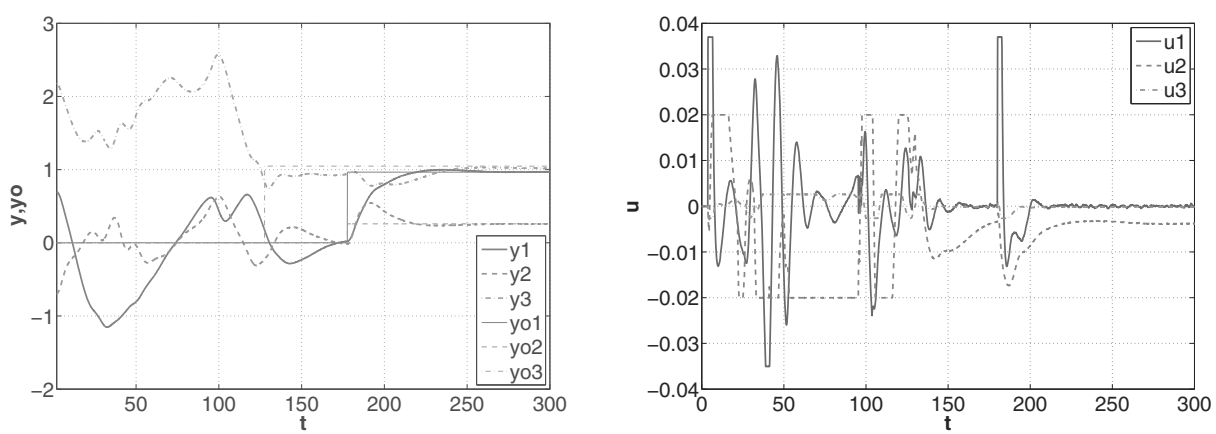

Fig. 9. Plots of controlled variables and control signals $\mathbf{u}(t)$ obtained for the ship's approaching the drilling point with the current.

use of a MIMO neural-modal controller is feasible. In contrast to stepwise tuned controllers, the responses of control signals $\mathbf{u}(t)$ are smoother and more possible to be realized by the propellers and the main engine 
of a real ship. The approximation of the surfaces by means of artificial neural networks provides "smoother" system operation (without any switching over) and also generalizes the parameter values of matrices $\mathbf{A}_{\text {ro }}$ and $\mathbf{B}_{\text {ro }}$ to untrained (unknown) values following from the quantization of signals $V_{s}$ and $\Psi_{c}-x_{3}(t)$ with an acceptable resolution, and eliminates the problem of an ambiguity in the operation of controllers, which assume different parameter values in the process of switching over depending on whether the auxiliary signals $V_{s}$ and $\Psi_{c}-x_{3}(t)$ increase or decrease.

\section{Acknowledgment}

The study has been partly supported by the grant no. $\mathrm{N}$ N514 679240 on Advanced control systems and algorithms for nonlinear dynamic MIMO plants financed by the Polish Ministry of Science and Higher Education.

\section{References}

Akesson, B. and Tojvonen, H. (2006). A neural network model predictive controller, Journal of Process Control 16(9): 937-946.

Åström, K. and Wittenmark, B. (1995). Adaptive Control, Addison Weseley, Reading.

Bańka, S. (2007). Multivariable Control Systems: A Polynomial Approach, Monographs of the Committee of Automation and Robotics, Polish Academy of Sciences, Szczecin University of Technology Press, Szczecin, (in Polish).

Bańka, S. (2012). On methods of modal controller synthesis in MIMO systems, in K. Malinowski and M. Busłowicz (Eds.), Advances in Control Theory and Automation, Printing House of Białystok University of Technology, Białystok, pp. 35-46.

Bańka, S., Dworak, P. and Brasel, M. (2010a). On control of nonlinear dynamic MIMO plants using a switchable structure of linear modal controllers, Pomiary, Automatyka, Kontrola 56(5): 385-391, (in Polish).

Bańka, S., Dworak, P., Brasel, M. and Latawiec, K.J. (2010b). A switched structure of linear MIMO controllers for positioning of a drillship on a sea surface, Proceedings of the 15th International Conference on Methods and Models in Automation and Robotics, MMAR 2010, Miedzyzdroje, Poland, pp. 249-254.

Bańka, S., Dworak, P. and Jaroszewski, K. (2011). Problems associated with realization of neural modal controllers designed to control multivariable dynamic systems, in K. Malinowski and R. Dindorf (Eds.), Advances of Automatics and Robotics, Kielce University of Technology Press, Kielce, pp. 27-41, (in Polish).

Bańka, S., Dworak, P. and Jaroszewski, K. (2013). Linear adaptive structure for control of a nonlinear MIMO dynamic plant, International Journal of Applied Mathematics and Computer Science 23(1): 47-63, DOI: 10.2478/amcs-2013-0005.
Bańka, S. and Latawiec, K.J. (2009). On steady-state error-free regulation of right-invertible LTI MIMO plants, Proceedings of the 14th International Conference on Methods and Models in Automation and Robotics, MMAR 2009, Międzyzdroje, Poland, DOI: 10.3182/20090819-3-PL-3002.00066.

Chen, J. and Yea, Y. (2002). Neural network-based predictive control for multivariable processes, Chemical Engineering Communications 189(7): 865-894.

Fabri, S. and Kadrikamanathan, V. (2001). Functional Adaptive Control. An Intelligent Systems Approach, Springer-Verlag, Berlin.

Khalil, H.K. (2001). Nonlinear Systems, Prentice Hall, Englewood Cliffs, NJ.

Ławryńczuk, M. (2010). Explicit neural network-based nonlinear predictive control with low computational complexity, in M. Szczuka, M. Kryszkiewicz, S. Ramanna, R. Jensen and Q. Hu (Eds.), Rough Sets and Current Trends in Computing, Lecture Notes in Computer Science, Vol. 6086, Springer, Berlin/Heidelberg, pp. 649-658.

Lee, C., Shin, M. and Chung, M. (2001). A design of gain-scheduled control for a linear parameter varying system: An application to flight control, Control Engineering Practice 9(1): 11-21.

Limon, D., Alamo, T. and Camacho, E. (2005). Enlarging the domain of attraction of MPC controllers, Automatica 41(4): 629-635.

Maciejowski, J. (2002). Predictive Control with Constraints, Prentice Hall, Englewood Cliffs, NJ.

Pedro, J.O. and Dahunsi, O.A. (2011). Neural network based feedback linearization control of a servo-hydraulic vehicle suspension system, International Journal of Applied Mathematics and Computer Science 21(1): 137-147, DOI: 10.2478/v10006-011-0010-5.

Qin, S. and Badgwell, T. (2003). A survey of industrial model predictive control technology, Control Engineering Practice 11(7): 733-764.

Rawlings, J. and Mayne, D. (2009). Model Predictive Control: Theory and Design, Nob Hill Publishing, Madison, WI.

Shevitz, D. and Paden, B. (1994). Lyapunov stability theory of nonsmooth systems, IEEE Transactions on Automatic Control 39(9): 1910-1914.

Tatjewski, P. (2007). Advanced Control of Industrial Processes, Springer-Verlag, London.

Tomera, M. (2010). Nonlinear controller design of a ship autopilot, International Journal of Applied Mathematics and Computer Science 20(2): 271-280, DOI: 10.2478/v10006-010-0020-8.

Tzirkel-Hancock, E. and Fallside, F. (1992). Stable control of nonlinear systems using neural networks, International Journal of Robust and Nonlinear Control 2(1): 63-86.

van der Boom, T., Botto, M. and Hoekstra, P. (2005). Design of an analytic constrained predictive controller using neural networks, International Journal of Systems Science 36(10): 639-650. 
Vidyasagar, M. (1985). Control System Synthesis: A Factorization Approach, MIT Press, Cambridge, MA.

Wise, D.A. and English, J.W. (1975). Tank and wind tunnel tests for a drill-ship with dynamic position control, Offshore Technology Conference, Dallas, TX, USA, pp. 103-118.

Witkowska, A., Tomera, M. and Śmierzchalski, R. (2007). A backstepping approach to ship course control, International Journal of Applied Mathematics and Computer Science 17(1): 73-85, DOI: 10.2478/v10006-007-0007-2.

Zhai, G. and Xu, X. (2010). A unified approach to stability analysis of switched linear descriptor systems under arbitrary switching, International Journal of Applied Mathematics and Computer Science 20(2): 249-259, DOI: 10.2478/v10006-010-0018-2.

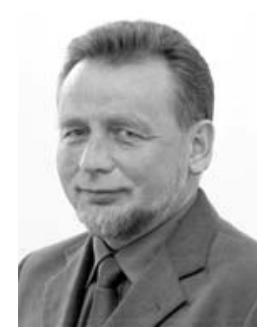

Stanisław Bańka received the M.Sc., Ph.D, and D.Sc. degrees in control engineering in 1969, 1976 and 1992, respectively. In the years 19962002 he was the dean of the Electrical Engineering Faculty at the Technical University of Szczecin and in 2003-2005 the director of the Institute of Control Engineering of that university. From 2009 a professor, then the head of the Department of Control Engineering and Robotics at the West Pomeranian University of Technology in Szczecin. Member of the Committee on Automatic and Robotics of the Polish Academy of Sciences (since 2003). Author of over 80 journal papers and three books. Main scientific interests: multivariable control systems, adaptive and optimal control, estimation and parameter identification, applications of informatics in automation. Professor Bańka passed away in February 2014.

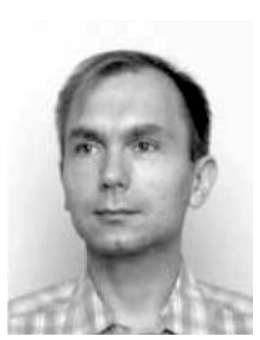

Pawel Dworak received the M.Sc. and Ph.D degrees in control engineering in 1999 and 2005, respectively. Currently an assistant professor at the Department of Control Engineering and Robotics, West Pomeranian University of Technology in Szczecin. Main scientific interests: multivariable control systems, adaptive control, process data acquisition and visualization, industrial applications of modern control algorithms.

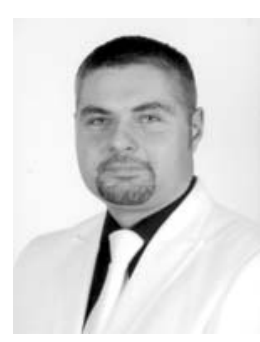

Krzysztof Jaroszewski received the M.Sc (2001) and Ph.D. (2007) degrees in control engineering. Currently an assistant professor at the Department of Control Engineering and Robotics, West Pomeranian University of Technology in Szczecin. Main scientific interests: artificial intelligence, especially neural networks, control systems and visualization, industrial diagnostic.

Received: 29 April 2013

Revised: 20 December 2013

Re-revised: 21 February 2014 